\title{
Nonlesional Focal Epilepsy: A Challenge from Genes to Surgery
}

Can J Neurol Sci. 2013; 40: 137-138

This month Nguyen and colleagues ${ }^{1}$ present the results of a retrospective study on the prevalence of nonlesional epilepsy in a tertiary epilepsy center.

The study's findings bring to light an especially difficult group of patients, from diagnosis to management. These authors show that one third of patients in a tertiary epilepsy center do not have any visible structural abnormalities on magnetic resonance imaging (MRI), and that half of these have pharmacoresistant epilepsy.

In this retrospective study many of the images were obtained with 1.5 Tesla MRI, which is associated with a lower sensitivity to detect small lesions. Strandberg and colleagues reported that in up to $20 \%$ of cases small lesions not visible with 1.5 Tesla MRI may be demonstrated with 3 Tesla MRI images ${ }^{2}$. But even if all patients had 3 Tesla MRI, which is currently clinically available in some epilepsy centers, approximately one quarter of adults with epilepsy would not have a clear structural epileptogenic lesion. This is a significant problem for patients with pharmacoresistant epilepsy, in whom epilepsy surgery may be the only way to manage seizures. Since no structural abnormalities are seen, many of these patients, especially those with extra-temporal lobe epilepsy, need further investigation in the form of intracranial electroencephalogram recordings in order to determine with precision the epileptogenic focus and to guide surgical planning.

Nonlesional epilepsy may be genetically determined, and most familial cases are not associated with any specific structural abnormalities. This is the case in autosomal dominant nocturnal frontal lobe epilepsy (ADNFLE). Luckily, most of these patients have seizures that can be easily controlled on carbamazepine. Different genes have been linked to $\mathrm{ADNFLE}^{3-9}$, but no specific gene or mutation is associated with structural abnormalities, nor can the genetic alterations predict which part of the frontal lobes will bear the epileptogenic focus. Therefore in the rare case of pharmacoresistant $\mathrm{ADNFLE}^{10}$, investigation with intracranial recording may be necessary.

On the other hand findings of structural abnormalities in familial mesial temporal lobe epilepsy are controversial. For instance one group found no abnormalities in 34 patients from 20 different families ${ }^{11}$ while another group found hippocampal atrophy in $57 \%$ of 84 patients (22 families) and 34\% of unaffected family members ${ }^{12,13}$. In both studies visual MRI inspection was done, however, quantitative hippocampal volumetry was also used in the Kobayashi studies. Although patients in the latter studies appear to have had more severe epilepsy, use of quantitative image analysis may, at least in part, explain the different results.

Quantitative measurements of volume and signal intensity can reveal abnormal epileptogenic tissue not previously detected on simple visual analysis. For instance, hippocampal or entorhinal cortex volumetry can point to the abnormal side in up to $25 \%$ of "image negative" temporal lobe cases ${ }^{14}$. Furthermore, when total volume quantification is not helpful, advanced quantitative methods based on surface shape models may point to the abnormal structure ${ }^{15,16}$.

In cases of focal cortical dysplasia, the abnormal lesion may not be seen on clinical MRI exams, even when obtained with higher field strengths. In such cases, identification of histologically proven focal cortical dysplasia may be increased by $30 \%$ if computer-based models of cortical thickness, blurring and tissue intensity derived from 3D T1 weighted sequences and combined into a single composite map are used ${ }^{17}$.

The study by Nguyen and colleagues demonstrates well the current limitations of MRI in focal epilepsy. As the authors point out, this knowledge alone should be of help to some nonlesional patients in their attempts to fully understand their condition. ${ }^{1}$

The findings also highlight the need to continue efforts to move special imaging techniques out of the labs and into the clinical setting. Imaging processing can reveal subtle abnormalities not previously identified by routine visual inspection and may ultimately lower the proportion of "image negative" cases of focal epilepsy. However, the various forms of imaging processing are highly specialized and time-consuming investigations, which are not routinely used and are performed mainly under investigation protocols paid for with research funds. It is not yet clear how much added benefit imaging processing will bring to the clinical setting, but this is a path that will certainly need to be followed in the coming years.

\author{
Danielle M. Andrade \\ University of Toronto, Toronto, Ontario, Canada
}

\section{REFERENCES}

1. Nguyen DK, Mbacfou MT, Nguyen DB, Lassonde M. Prevalence of nonlesional focal epilepsy in an adult epilepsy clinic. Can J Neurol Sci. 2013;40(2): 198-202.

2. Strandberg M, Larsson EM, Backman S, Kallen K. Pre-surgical epilepsy evaluation using 3T MRI. Do surface coils provide additional information? Epileptic Disord. 2008;10:83-92.

3. Scheffer IE, Bhatia KP, Lopes-Cendes I, et al. Autosomal dominant nocturnal frontal lobe epilepsy. A distinctive clinical disorder. Brain. 1995;118(Pt 1):61-73.

4. Phillips HA, Scheffer IE, Berkovic SF, Hollway GE, Sutherland GR, Mulley JC. Localization of a gene for autosomal dominant nocturnal frontal lobe epilepsy to chromosome $20 \mathrm{q}$ 13.2. Nat Genet. 1995;10:117-18.

5. Phillips HA, Scheffer IE, Crossland KM, et al. Autosomal dominant nocturnal frontal-lobe epilepsy: genetic heterogeneity and evidence for a second locus at 15q24. Am J Hum Genet. 1998; 63:1108-16. 
6. Phillips HA, Favre I, Kirkpatrick M, et al. CHRNB2 is the second acetylcholine receptor subunit associated with autosomal dominant nocturnal frontal lobe epilepsy. Am J Hum Genet. $2001 ; 68: 225-31$.

7. De Fusco M, Becchetti A, Patrignani A, et al. The nicotinic receptor beta 2 subunit is mutant in nocturnal frontal lobe epilepsy. Nat Genet. 2000;26:275-6.

8. Gambardella A, Annesi G, De Fusco M, et al. A new locus for autosomal dominant nocturnal frontal lobe epilepsy maps to chromosome 1. Neurology. 2000;55:1467-71.

9. Leniger T, Kananura C, Hufnagel A, Bertrand S, Bertrand D, Steinlein OK. A new Chrna4 mutation with low penetrance in nocturnal frontal lobe epilepsy. Epilepsia. 2003;44:981-5.

10. Derry CP, Heron SE, Phillips F, et al. Severe autosomal dominant nocturnal frontal lobe epilepsy associated with psychiatric disorders and intellectual disability. Epilepsia. 2008;49:2125-9.

11. Crompton DE, Scheffer IE, Taylor I, et al. Familial mesial temporal lobe epilepsy: a benign epilepsy syndrome showing complex inheritance. Brain. 2010;133:3221-31.

12. Kobayashi E, Li LM, Lopes-Cendes I, Cendes F. Magnetic resonance imaging evidence of hippocampal sclerosis in asymptomatic, first-degree relatives of patients with familial mesial temporal lobe epilepsy. Arch Neurol. 2002;59:1891-4.
13. Kobayashi E, Lopes-Cendes I, Guerreiro CA, Sousa SC, Guerreiro MM, Cendes F. Seizure outcome and hippocampal atrophy in familial mesial temporal lobe epilepsy. Neurology. 2001;56: 166-72.

14. Bernasconi N, Bernasconi A, Andermann F, Dubeau F, Feindel W, Reutens DC. Entorhinal cortex in temporal lobe epilepsy: a quantitative MRI study. Neurology. 1999;52:1870-6.

15. Hogan RE, Carne RP, Kilpatrick CJ, et al. Hippocampal deformation mapping in MRI negative PET positive temporal lobe epilepsy. J Neurol Neurosurg Psychiatry. 2008;79:636-40.

16. Kim H, Besson P, Colliot O, Bernasconi A, Bernasconi N. Surfacebased vector analysis using heat equation interpolation: a new approach to quantify local hippocampal volume changes. Medical image computing and computer-assisted intervention: MICCAI International Conference on Medical Image Computing and Computer-Assisted Intervention. 2008;11: 1008-15.

17. Bernasconi A, Antel SB, Collins DL, et al. Texture analysis and morphological processing of magnetic resonance imaging assist detection of focal cortical dysplasia in extra-temporal partial epilepsy. Ann Neurol. 2001;49:770-5. 